\title{
Measuring System of Vibration Diagnostic with the Metrological Self-Control Function
}

\author{
A. Grigoriev, S. Lebedev, A. Timofeev
}

\begin{abstract}
In the article, there is given the description of the method of metrological self-control in the measuring system of vibration diagnostic, the structural system of scheme is shown and the peculiarities of its functioning is self-control mode are given. The diagnostic methods, discussed in the article, usually are not completely independent and show the bigger effectiveness in the combined usage. The following problem exists: in the early stages of the appearing of a defect the informative components of vibration signals have extremely small amount of the energy and are covered with the background noise. Therefore, the effective method of the signal processing should assume the extraction of the informative signs of a damage when the signal/noise ratio is less than 1. Currently the biggest interest is presented by the group of the time frequency methods because they allow localizing in time the peculiarities of a vibration signal, therefore, they are potentially more sensitive to occurring defects than the time and spectral methods. Among their disadvantages are the mathematical complexity and the complexity of the realization and the interpretation of the results. The main advantage of developing system is the presence of the high frequency vibration measurement channel and also the built-in functional self-diagnosis system which principle is experimentally confirmed.
\end{abstract} processing, vibration diagnostics, vibration sensor.

\section{INTRODUCTION}

Nowadays technical systems and equipment contain many integrated sensors providing management and diagnostic. The traditional methods of control metrological serviceability of the sensors themselves namely checking or calibration at the intervals 1-2 years are not economically effective now. The traditional methods of control are changed into alternative methods of self-control that are provided by [1]:

- lowing the risk of appearing undetected metrological failure;

- grounding of assigning the inter-calibration interval depending on residual metrological resource;

- lowing the operational expenses due to reduction of quantity of checking and calibration;

lowing the losses connected with the metrological refusal consequences.

Revised Manuscript Received on October 30, 2019.

* Correspondence Author

Anatoly Grigoriev, LLC SensFab GmbH, Dortmund, Germany. Email: prof.agri@gmail.com

Sergey Lebedev*, Design Center "Design of integrate microelectronic system", National Research University of Electronic Technology (MIET), Moscow, Russian Federation. Email: lebedevsergey11@ rambler.ru

Alexey Timofeev*, Department of measurement, Joint Stock Company "Zelenograd nanotechnology center", Moscow, Russian Federation. Email: alex_93_ti@mail.ru
Keywords: Metrological self-control, microcircuit, signal

Beginning from 2000's in the world the intensifying of works on the implantation of the automatic control of the metrological serviceability (self-control) of the sensors in the process of exploitation is growing. This fact confirms the creation of specialized sections on the conferences and symposia «Sensor-2005» in Nuremberg (Germany), ISMTII 2007 Sendai (Japan) on this issue [2].

For increasing the time intervals between the technical services and transition from the periodic schedules of technical services to the technical services on necessity it is needed to create the system of monitoring the state of work nodes. Such systems, using the complicated data processing algorithms, can warn on the occurring problems and the necessity of preventive maintenance or repair. The implementation of such devices promotes increasing the level of reliability and safety in the operation of the complex technical systems and also allows reducing the repair costs during the whole period of product exploitation.

For solving the question of constant monitoring of the state of circling object with the help of the sensor of vibration the special hardware development is needed. In the result of analysis of the sources it has found out that it is necessary to use two channels of measuring the vibration data (using one channel leads to a huge discretization frequency and very big dynamic range at the same time). The frequency ranges of both channels were chosen, the dynamic range of each channel and the basic requirements for the signal conversion and processing are presented. Wherein the optimal bit depth analog-to-digital converter (ADC) and the separation of the functions between the analog and digital data processing subsystems have been identified. For simplifying the process of the mounting vibration sensor and reducing the cost of its production the integrated version of analog and digital parts in the shape of microcircuits with the possibilities of the connection of external sensitive elements of a bending type (piezo resistive beam accelerometers) is selected.

For use in the highly loaded drives and the turbines of general purpose there is needed the device that will provide the continuous monitoring of the state of moving objects and also recording the emergency situations with saving the work reports into a constant non-volatile memory for the further analysis in case of the appearing emergency situations.

\section{A.The structure of vibration sensors}

The standard structure of the vibration sensor includes the following blocks: the sensitive element, the analog filter unit, the amplifier, the analog-to-digital converter and the microcontroller, the communication interface.

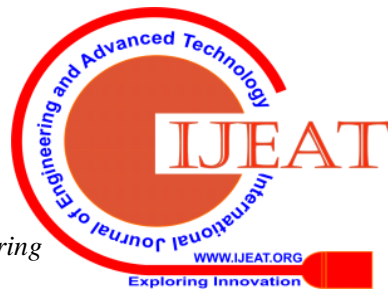




\section{Measuring System of Vibration Diagnostic with the Metrological Self-Control Function}

The realization of this structure in a single housing of the built-in sensor will demand solving the row of tasks starting from the spatial arrangement of the sensitive elements and other components inside the case to testing the technology of serial assembly of sensors.

One of the most widely spread types of the sensitive elements, being used for converting the vibration into electrical signal, is, nowadays, the piezoelectric accelerometers. Such sensors have the compact geometric dimensions and low weight. The market of the accelerometers is conditionally divided into two types: the piezoelectric and micro-electromechanical (MEMC) accelerometers. For measuring the high-frequency vibration acceleration MEMC-sensors are unsuitable because have their own oscillation frequency in the range from 10 to $30 \mathrm{kHz}$. For the low frequency measurements, the piezoelectric sensors are unsuitable because their working range starts from $500 \mathrm{~Hz}$. So, for example, there are vibration sensors for the low frequency measurements produced by PCB PiezotronicsInc (USA), but their range is limited only by $25.4 \mathrm{~mm} / \mathrm{s}$ [2].

\section{B. The use of the accelerometers}

Aiming to cover both ranges of the frequencies, for measuring the vibration wide bandwidth accelerometers are used. Presently all the wide bandwidth accelerometers are the compensation type accelerometers [3] both axial and pendulum $[4,5]$. The sensors by AnalogDevices of ADIS1 series have a bandwidth up to $14.24 \mathrm{kHz}$, Colibrys (Switzerland) VS9000 - up to $3 \mathrm{kHz}$. So, such companies that are well known by their accelerometers, as STMicroelectronics, Freescale Semiconductor, Murata do not produce the sensors suitable for these options [6].

For measuring the vibration by the most rational choice the piezoelectric accelerometers with a wide frequency range, good sensitivity and resolution which, besides it, are easy to install, are used [7].

MEMC-sensors have been successfully implemented in the monitoring system of the high speed trains ICE. Siemens also developed a monitoring system for the wagon trolleys that determines the wear of the various knots of the carts: the bearings, shafts, brakes, wheels - and on the basis of the received information reveals the potentially possible defects, capable of provoking an accident. In this system they use 24 sensors Colibrys on one railway carriage and about 200 sensors for the whole train. The first three trains were created in 2010 and the final certification passed in 2011.

The company Colibrys used the other way in sensor VS9000 D. According to the manufacturer, the sensors with the variable capacity VS9100.D can advantageously replace the traditional piezo-resistive solutions in many applications. For the given range of the acceleration and frequency bands the capacitive sensors provide lower noise and much more long-term and temperature stability. Structurally the sensors VS9000.D represent themselves three silicon wafers, with a differential change in capacitance between them. The suspension stiffness can be changed by the constant voltage on the capacitive plates.

\section{C.The A/D Converters}

The demanding bit depth of the A/D converter (A/D C) for a built-in intelligent vibration sensor is calculated basing on the parameters of the minimum and maximum signal amplitude. The RMS vibration velocity range for the developing sensor is $0.1-226 \mathrm{~mm} / \mathrm{s}$ in the band $5-1000 \mathrm{~Hz}$. If to use the A/D converter for the whole range of the vibration speed then the necessary bit depth will be on the level of 18-19 bit. Using two ranges of sensitivity, for example from 0.1 to $12 \mathrm{~mm} / \mathrm{s}$ and from 12 to $226.24 \mathrm{~mm} / \mathrm{s}$, this method will allow not only reducing the demands to the sensitivity element of the sensor but also using the ADC with the bit depth on the level of 14-16 bit. This substantially makes the construction of the sensor cheaper as on developing the principle scheme as well as on the integrated circuit design.

In the most of the existing on the market integrated analog input paths the ADC with the bit depth not less than 24 bit and the reasonably slow sampling rate are used. For example, the integrated analog input path TexasInstruments ADS1293 has the quite big digitization speed, because they are used in the cardiac recorders. However, with the increasing of the sampling frequencies the RMS of the RMS value of the noise increase abruptly: from $0.95 \mu \mathrm{V}$ at a sampling frequency of $100 \mathrm{~Hz}$ to $4.74 \mu \mathrm{V}$ at a sampling frequency of $3.2 \mathrm{kHz}$. Such abrupt noise increasing does not allow talking about the effective 24 bits ADC.

The ADC with the sampling frequency of at least $4-8 \mathrm{kHz}$ is needed in a vibration sensor. On the market nowadays there are presented the high-grade ADC only for picking up and processing the slow signals (the temperature, pressure etc.). Therefore, the development of the technology for the production of a device of this type is a perspective task.

The usage of the developing device is supposed at the railway and industrial facilities for measuring the vibration parameters of the most critical nodes with the environment temperature from -40 up to $+125^{\circ} \mathrm{C}$. The impact of heightened temperature leads to the shortening of the electrical component service life. According to the data of the company Analog Devices, the time of MTBF for the precision instrumentation amplifier AD8229 [8] is 20 thousand hours in $120^{\circ} \mathrm{C}, 10$ thousand hours in $140{ }^{\circ} \mathrm{C}, 5$ thousand hours in $165^{\circ} \mathrm{C}$. The important factor is the changing of properties of the electronic components, because most of the microcontrollers consume, on the average, $10-15 \%$ more energy in increasing of the environment temperature than on $40^{\circ} \mathrm{C}$ from the normal conditions with the same calculating loads. Also, it is needed to take into account the increase of the noise level with the temperature increasing. For AD8367 made by Analog Devices [9] the level of noise increases on $13 \%$ with the temperature increasing from 25 to 85 .

\section{D.Microcontrollers}

The calculating system core represents by itself the microcontroller, performing the tasks of digitizing analog signals, the additional transformations, parameter calculations, and also communicates with the interrogating external system, provides the stable work of the sensor and saves the calibration factor information and bounce logs. Choosing the microcontroller first of all such parameters as the speed, memory volume, the presence of developed peripherals, the presence of the built-in ADC and its capacity are important. Nowadays the powerful microcontrollers on the base core ARM and Cortex are developed, the calculating resources of such controllers are almost on the same level

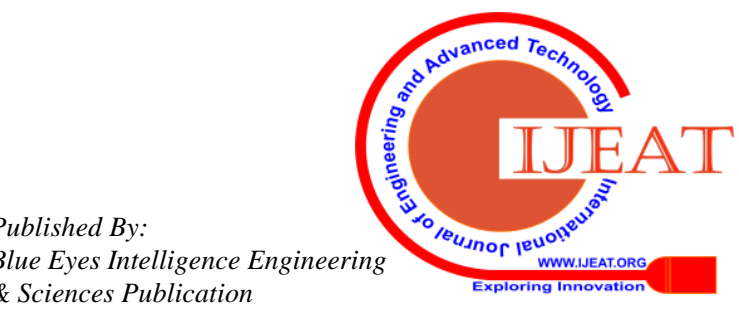


with the one's of PC and specialized devices. It's useful to note, that the growth of productivity does not entail the proportional increase in the chip cost, so, for example, the cost of powerful 32-bit core-based microcontroller Cortex-M3 is compatible to the cost of 16-bit mixed signal microcontroller MSP430. Besides it, there are the hybrid microcontrollers with an additional core; it allows using the basic functions of the digital signal processing without complicating the sensor hardware. These trends will continue to develop, because, for example, the company ST Microelectronics purposefully supports the maximum compatibility of the microcontroller families on the output and strapping that allows raising the productivity of a system with the release of a new more powerful microcontroller without changing the hardware.

The Analog Filter Unit can be very simple for example in the pre-shaping the signal band or may have a complicated device with the changing coefficients and the supply of control signals from the calculating core.

The most critical parameters for the input amplifier there are the input resistance value, the noise level in a given frequency band, the common mode interference of the rejection level. The value of the input impedance first of all influences upon the lower bound of the useful signal frequency bands. The latest tendencies of the instrumentation amplifiers development show the stable growth of the input impedance, for example, for AD627 [10] this parameter stands on the level $20 \mathrm{GOm}$ at $2 \mathrm{pF}$, and for the more modern instrumentation amplifier AD8221 it is $100 \mathrm{GO}$ at $2 \mathrm{pF}$ [11]. Moreover, these amplifiers belong to the same manufacturer classification segment. The second important parameter of the input amplifier is the level of noise in the desired frequency band. So, for example, for the mentioned above amplifier AD627 the noise on the frequency $1 \mathrm{kHz}$ is $38 \mathrm{nV} / \mathrm{Hz}$, on the much lower frequencies the influence of, so called, the flicker noise or noise $1 / f$ appears; this noise appears most of all on the low frequencies - the production company adduces the values $48-50 \mathrm{nV} / \mathrm{Hz}$ at $1 \mathrm{~Hz}$.

\section{E.Operational Amplifiers and Transceivers}

The instrumentation amplifiers can be replaced with the standard operational amplifiers (OA), in the case if the impact of the interference on the input stage will be minimal (for example, if sensor housing is reliable screen). The usage of the low noise OA allows reducing significantly the impact of the input noise because this parameter of OA is significantly lower than the same one of the instrumentation amplifiers. So, for example, for OA AD8606 the noise level is $8 \mathrm{nV} / \mathrm{Hz}$ comparing to $38 \mathrm{nV} / \mathrm{Hz}$ of $\mathrm{AD} 627$.

The connection interface represents the transceiver (for example the interface RS-485) or the 4-20 mA current line generator. The development of the electronic components in this sphere mostly comes on the following direction: the increasing in the number of connected nodes in the network (for RS-485), the lowing of the power consumption, increasing the reliability of the galvanic upshot. Currently there are the transceivers RS-485 with the quantity of the nodes in the network up to 256 (the further increasing of numbers of the nodes is impractical because of the specification of the exchange protocol), and the galvanic upshot from 2.5 to $5 \mathrm{kV}$.

The demands to the power transceivers are also very important because the microcontrollers basically have the supply voltage $3.3 \mathrm{~V}$, accordingly, the input / output lines of the transceivers should supply the allowable voltage levels. In the past the standard supply for these components was the level of voltage $5 \mathrm{~V}$ that leaded to including the additional level converters. Now there is a big choice of the transceivers with a supply voltage of $3.3 \mathrm{~V}$, though and the microcontrollers more often are created with the outputs tolerate to $5 \mathrm{~V}$ power levels.

More often the choice of the components for the power supply sub-system is based on the noise level on the output and component reliability. Also, the important criteria are the power dissipation because in the case of using in the electronic unit of the precision amplifiers - the local board warming may lead to the substantial denominations of the amplifier circuit's passive components.

\section{F. The choice of the electronic components in the creating small-sized vibration sensor}

On the developing small-sized vibration sensor it is necessary to think about the possible variants of using the more expensive component with the much smaller space on a circuit board or in a sensor housing volume. So, for example, previously for the vibration sensors there were used the sensitive elements in a shape of a plate or disc with the sizes comparable with the sizes of a circuit board with the electronic components (the diameter around 25-30 mm). The substantial contribution into the reduction in the overall dimensions of the vibration sensors was the transition on the other kinds of piezo-ceramic sensitive elements such as a beam type element. This species of the sensitive element is significantly more complicated in the production but for this system the price can be neglected. The advantage of this element is that it can be placed on the printed circuit board itself or next to the micro assembly.

Choosing the electronic components for the sensor the changes of the ratings of the passive components also should be taken into account especially it concerns the capacitors. Usually the precision and thermostable components have the bigger size than the common consumption components. One of the variants of the economy of the space on a circuit board can be accurate and detailed calculation of every component applicability. On the developing of the totally integral vibration sensor the maximum capacities and the type of material, used in the integral scheme, should be considered.

The terms of use of the sensor also impose the certain restrictions on the choice of the element base and the electronic units producing technology. The direct influence of the temperature on the components characteristics was reviewed above; however, there is a list of the indirect factors. For example, using the sealing compound, the temperature changes can cause the compression or expansion effects because most of the plastic compounds have the big thermal expansion coefficients sometimes leading to a wire breakage. It leads to the strong squeezing and bending of the printed circuit boards of the electronic unit. The compact sizes of the sensors do not allow using the output components and all the elements of the surface mounting cannot cope with the mechanical voltage. Raising the possible level of the voltage can be done with the help of the soldering technology with using the conductive glue during the mounting (such glue is more plastic than the standard kinds of soldering).

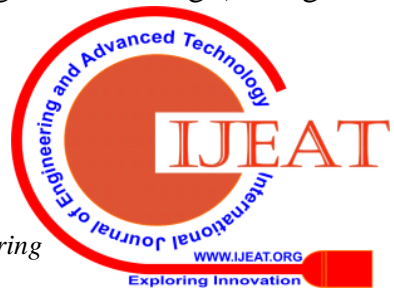




\section{Measuring System of Vibration Diagnostic with the Metrological Self-Control Function}

The background vibrations also may lead to the deformations and damage of the electronic components. To raise the resonance frequencies of the electronic units can be constructive or using the varnishing components, the compounding also can protect the components of the surface mounting as well.

\section{METHODOLOGY}

\section{A.The methods of vibration diagnostics}

For finding out and identification of the defects there is a big variety of the diagnostic methods based on the analysis of the vibration signals. Contingently these methods can be divided into three basic categories: the time, the frequency and the time-frequency [12].

The time methods, as a rule, are used for the preliminary defect detection; for its identification there is a necessity of the vibration signal spectrum analysis. The defects of the different nature are demonstrated differently in a spectrum but to find them out is possible only in a case of their significant severity and periodicity because otherwise the energy of their relevant spectrum components will be less than the background noise.

The time-frequency methods are used mainly for the processing and analysis of the nonlinear and non-stationary signals. The usage of these methods in vibration diagnostic allows finding out the defects of the rolling elements in the earlier stages due to the localization possibility of the analyzing signal peculiarities that are, as a rule, the indicators of the appearing defect.

The methods on the amplitude spectrum cepstrum analysis (the power cepstrum technique) or the vibration envelope spectrum belong to the frequency methods.

The method of analysis on the amplitude spectrum allows finding out the amplitude bursts, that are periodical, in a vibration signal, moreover, there is the unequivocal match between the periods of bursts and the defect nature. In this way, the presence of the vibration signal in the spectrum points about the defect and its amplitude says about its severity. Using this method, it is possible to find out the following defects: the imbalance loose bearing, grazing the rolling elements in bearing and seals, increased clearance in bearings, running in outer ring [13]. The advantages of this method are in its high information content and noise immunity. The diagnostic parameters in this case, there is the vibrational spectral density $S(w)$, formula (1):

$$
S(w)=\int_{0}^{T} s(t) e^{-j w t} d t
$$

where $e^{-j w t}$-complex frequency function; $T$ - time interval. The method of analysis based on the energy spectrum is similar to the previous one but the diagnostic parameter is the energy spectrum of the signal $G(w)$, formula (2):

$$
G(w)=\frac{|S(w)|^{2}}{2 \pi}
$$

The cepstrum analysis is similar to the Fourier's direct transformation from the logarithmic spectrum of a signal power; it allows finding out the periodical structures in a signal spectrum $C(q)$, formula (3):

$$
C(q)=\left|F\left\{\log _{2}[G(w)]\right\}\right|^{2}
$$

where $F-$ Fourier's transformation.

The cepstrum analysis allows finding out the defects of the bearing outer circle [14] and is characterized by the high resolution.

Using the spectrum analysis method of the vibration signal envelope the following defects can be found out: the skew of the outer ring on the grounding, the heterogeneous radial stretch, the slipping into the seat, the deterioration of the rolling element surface, the surface friction group defect, the chipped inner and outer circles, the running in the outer circle. This method is characterized by the high sensitivity and allows finding out the defects on the early stages.

\section{B.Amplitude modulation of a vibration signal}

The presence of the bearing defects causes the amplitude modulations of a vibration signal. The frequency of the modulation is determined by the type of a defect; the depth of the modulation is determined by the size of a defect. The spectrum of the envelope is defined in the whole vibration signal with the help of a narrow band filter; then the demodulation should be done.

The diagnostic parameters in this method there are the amplitudes and frequencies of harmonious envelope spectrum components. The defect of the friction surface leads to the vibration modulation with the certain frequency $f_{i}$, in the spectrum harmonious components with the divisible $f_{i}$ frequencies also appear. The frequency $f_{i}$ and the number of the divisible harmonics define the defect type and the degree of defect appearing can be estimated by the level difference of the maximum harmonic and the background.

\section{C.Metrological self-control method}

On the projecting modern intellectual measuring systems, it is needed to foresee the possibility of the metrological self-control sensors according with [15]. The metrological self-control provides the estimation of the metrological intellectual sensor work or the intellectual measuring system with the definition of the measuring result status. The metrological self-control should be based on the additional data, getting on due to the space (structural), time, informational (functional) redundancy that is or has formed in a sensor or measuring system. The volume of the additional data can be increased by the combination of these kinds of the redundancies.

As a method of self-control, the direct method of metrological self-control, based on the structural redundancy, was chosen. This method allows proving the self-control and diagnostic of the sensor and processing signal units' mistakes and also estimating the reliability of getting the information about the vibration parameters.

Besides the main measuring channel there is also additional diagnostic channel that forms the basic values of the frequency and vibration signal amplitude. 
In the process of self-control, the measured parameters are compared on the main channel and the supporting one. In the case of the inconsistencies of the given parameters, the measuring system is defined as inoperative.

\section{D.Sensor data defining algorithm and their verification}

Denote the data massif of the vibration acceleration having got from the rolling bearing after ADC digitization as $p(t)$, where $t$ - time measured in the seconds. Introduce also a diagnostic signal of a set frequency $\omega_{d}$, that denote as $d(t)$. The super position $p(t)$ and $d(t)$ denote as $f(t)$.

Then in the software MATLAB the frequency analysis will be done $f(t)$ through the algorithm of Fourier fast transform $f$ (FFT), Goertzel and the filtration in the frequency range up to $1 \mathrm{kHz}$. The same algorithms of processing are implemented in the developed chip "VIBRO-ASIC". In this way the massif $f(t)$ is converted into a spectral density function $S(\omega)$, where $\omega$ - harmonics' frequency in Hz. To visualize the results the spectral density distribution schedule $S(\omega)$ should be built from which the frequency values $\omega_{1}, \omega_{2}$ with the maximum amplitude will be found out. In the case if one of the frequency values $\omega_{1}$ or $\omega_{2}$ matches the frequency of the diagnostic signal $\omega_{d}$ it may be concluded as the adequacy of the obtained vibration acceleration data of the rolling bearing. If there are no such a match it is needed to make re-measurement or define the system inoperative.

\section{RESULTS}

\section{A.The system of vibration diagnostics based on the specialized microcircuit "VIBRO-ASIC"}

The developed system of vibration diagnostics based on the specialized microcircuit "VIBRO-ASIC", that is compatible with the piezo-ceramic sensors of the capacitive type, Fig. 1. The advantages of the integral performance are in connected with the reducing the overall dimensions of the system that allows putting the device not far from the diagnosed objects and also in the reducing the market price.

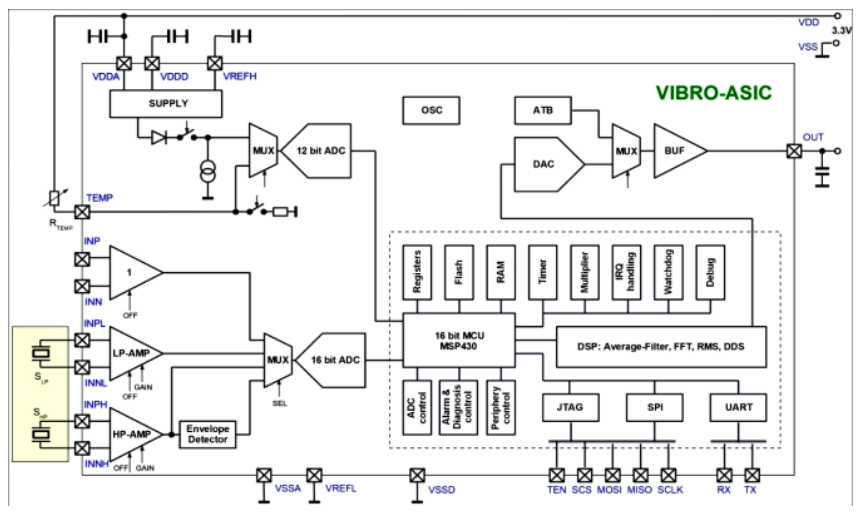

Fig. 1. The structural scheme of the vibration diagnostic system based on the specialized microcircuit "VIBRO-ASIC"

The analog part of the microcircuit "VIBRO-ASIC" consists of the output channels: changing the vibration (the low and high frequency) and the temperature. The digital part includes: the 16-bit microcontroller, the signal processor and the interface unit (SPI, UART, JTAG).

The microcircuit includes a 16-bit analog-to-digital converter (ADT) of the consecutive approximation with a

conversion time of $2 \mu \mathrm{s}$, that correspond to the frequency sampling of $500 \mathrm{kHz}$. The outputs of ADC are connected via the multiplexer to the outputs of instrumental amplifiers of the low- and high-frequency channels. The ADC was made on the base of the 16-bit differential capacitive matrix with the isolation capacitor; it works on the principle of the consistent approximation and is calibrated via the test interface JTAG.

The low-frequency channel contains the instrumental voltage amplifier with the high input impedance and the programed amplification coefficient from 2 to 104, with the fixed upper bandwidth $10 \mathrm{kHz}$.

The high-frequency channel includes the same voltage amplifier but with the bandwidth from 28 to $32 \mathrm{kHz}$.

The integrated circuit clock works on the calibrated frequency $16 \mathrm{MHz}$ with the accuracy $\pm 2 \%$ and the possibility of its trimming in the wide range in the increments of no more than $1,5 \%$, that is demanded by the asynchronous interface accuracy requirements UART.

The interface JTAG is intended for making the laboratory measurements and testing the microcircuit with the possibility of the access to its all functional blocks.

In the context of the systems of the vibration diagnostics, the sensitivity element may be made on the base of the piezo-ceramics. The output signal of the piezoelectric vibration transducer is the electric charge accumulated in it, that in a working frequency range is proportional to the acceleration making on the sensor. This charge is created in the basic sensor plate (or the group of the plates), made of the piezoelectric material on its deformation. There is a cargo of known mass $m_{\text {sens }}$ is attached. With the acceleration $a_{\text {lim }}$ force acts on the plate (4):

$$
F_{\text {seg }}=a_{\text {lim }} \cdot m_{\text {sens }}
$$

where $F_{\text {seg }}$ - acting force.

Under the action of this force the charge is created the size of which depends on the using material. As it has been said, in the working range this charge is proportional to the acting force as well as the acceleration. Usually the sensors are characterized by the conversion coefficient $K_{S}$, that connects the charge and acceleration (5):

$$
Q_{s}(t)=K_{s} \cdot a(t)
$$

where $Q_{s}(t)$ - is the charge generated in the sensor.

Based on the chosen self-control method the block of the functional self-diagnostic was added to the structural scheme on the Fig. 1, Fig. 2.

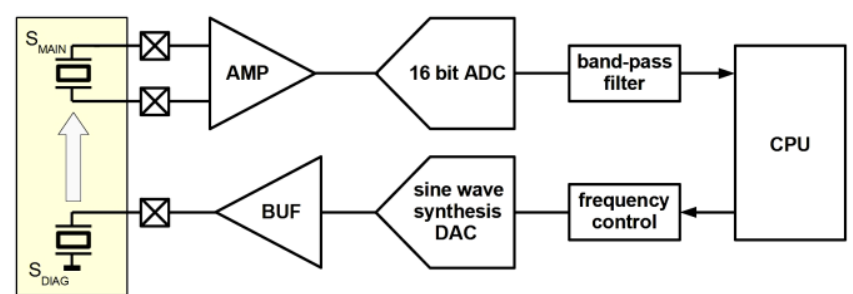

Fig. 2. The structural scheme of functional self-diagnostic block 


\section{Measuring System of Vibration Diagnostic with the Metrological Self-Control Function}

\section{B. Metrological check of the sensor serviceability}

For checking the metrological serviceability of the sensor (sensors), that is the part of measuring system, the additional data is used, getting due to the structural system redundancy. The structural redundancy is supplied by the usage of the additional sensitive piezoelectric element in the system installed directly next to the basic sensor. The additional sensitive piezo-element, using as a source of the vibration signal looks like an ordinary piezo-sensor with the characteristics close to the same piezo-sensor.

On the Fig. 2 there is a block-scheme of the basic and additional measuring channels. The additional channel contains the harmonic signal synthesizer managed by the processor. In the test mode, the microprocessor activates the synthesizer of harmonic signal, which signal goes through the digital to analog converter (DAC) and the outer buffer to the additional piezo-element that, in its turn, generates the diagnostic vibration signal for the basic piezo sensor. After processing the diagnostic signal, the system makes a decision about the efficiency of the measuring system. DAC of the system of the metrological self-diagnostic is produced on the non-linear resistive principle and is formed by the sine wave on 12 points, Fig. 3 and 4 .

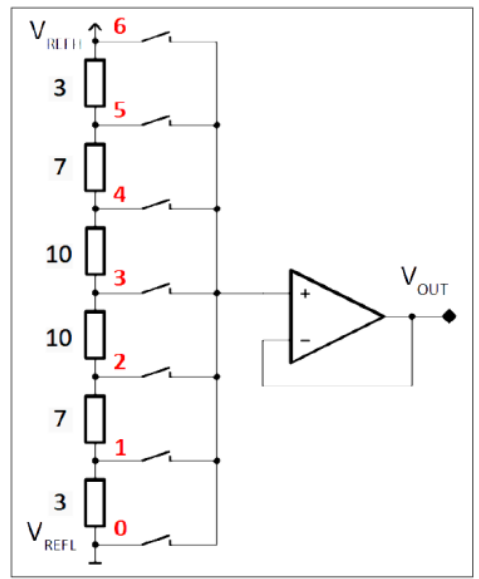

Fig. 3. The scheme of the resistive DAC of the system of metrological self-control

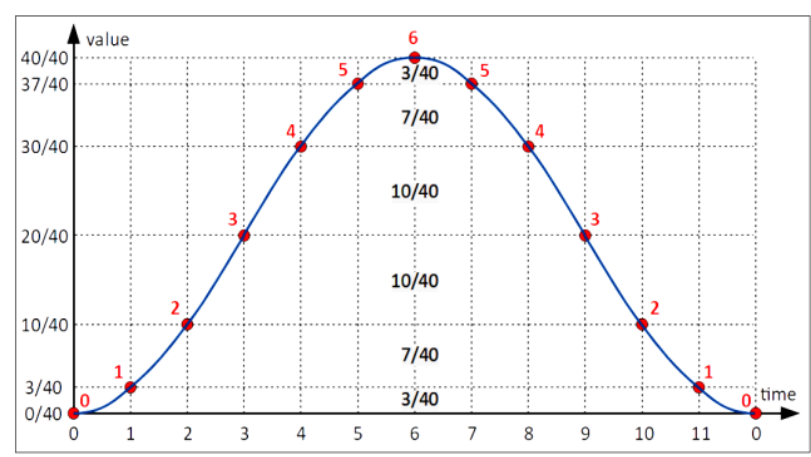

Fig. 4. The schedule of the forming the self-control metrological system of harmonious signal

The measurements are shown in the real time when all the piezo-electric elements are settled on the vibrating object. The self-control system tasks consist in the advanced selected known synthesized signal of the set frequency on the noise background and in the advanced unknown useful signal of the main sensor.

As a reference value it is used the measurement of the vibration signal, controlled by the transducer on exposing of an additional piezo-electric element that is defined by the previous calibration.

By deviation of the ratio of values of outer signals, measured in the process of the exploitation, from the reference value the metrological sensor serviceability may be estimated [13-18].

\section{C.The experimental verification of the self-control system}

For checking the principles of work of the self-control system the following experiment was held. The data of the vibration acceleration, taken from the rolling bearing with the help of the system model, containing the analog blocks are the piezoelectric sensor, preamplifier with the measured bandwidth and the 16 bit ADC with the frequency of sampling $250 \mathrm{kHz}$, were processed in MATLAB in complete accordance with the digital processing algorithms in the microchip "VIBRO-ASIC".

The result of the work of the system of the self-control on the real signal of the rolling bearing is given on the Fig. 5-9. On the Fig. 5 there are the digitalized inner signals with the off and on self-control system and on the Fig. 6, accordingly, there is the calculated spectral density of the same signals.

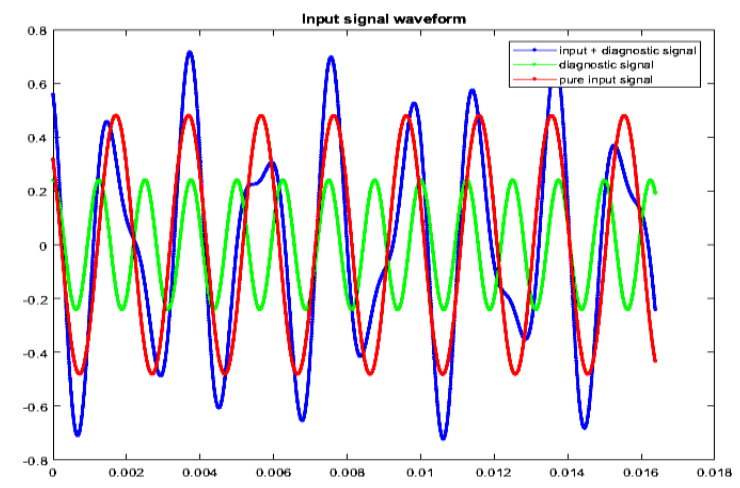

Fig. 5. The digitized outer signal with the activated self-control system

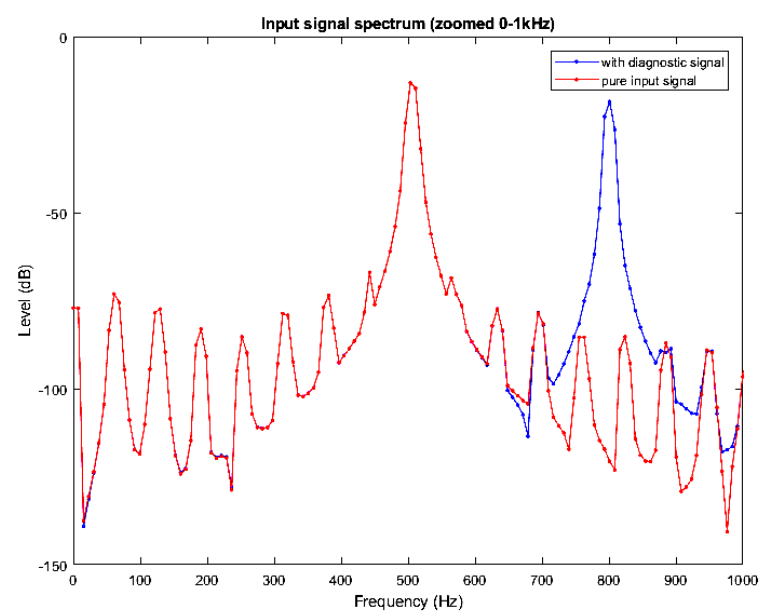

Fig. 6. The spectral vibration acceleration density of the inner signal

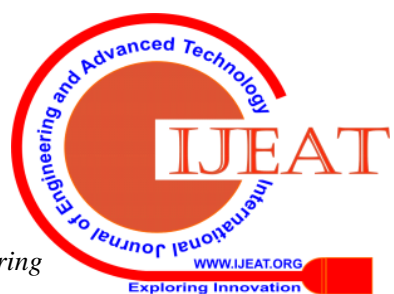


On the Fig. 7, there are the signals after $1 \mathrm{kHz}$ filtration with the turned off and on self-control and on the Fig. 8, accordingly, there is the calculated spectrum density of the same signals.

On Fig. 9, there is the spectral density calculated on the FFT algorithm with the turned off and on self-control system. In the microchip "VIBRO-ASIC" there are used 4 independent configurable Goertzel’s blocks, named Block №1 - Block №4.

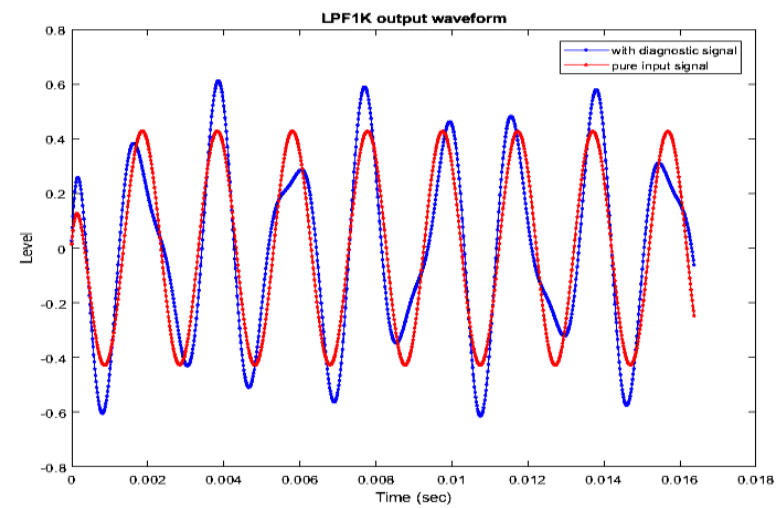

Fig. 7. The signal after $1 \mathrm{kHz}$ filtration with the activated system of self-control

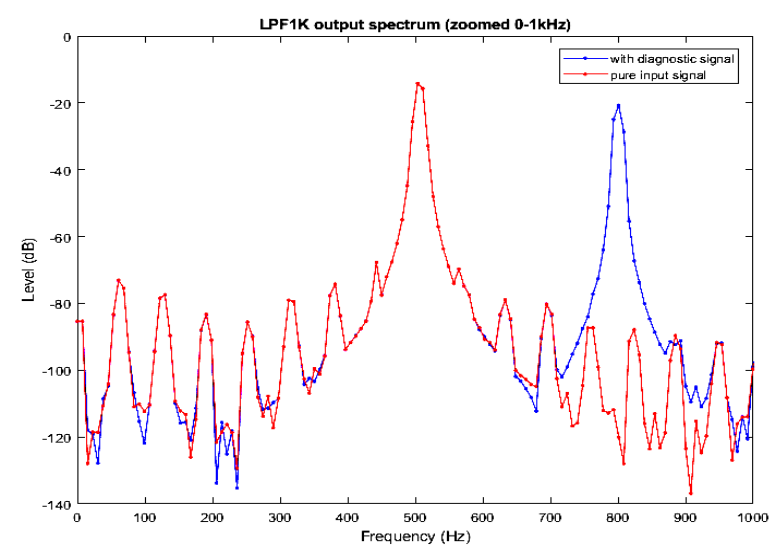

Fig. 8. The spectral density of a signal after $1 \mathrm{kHz}$ of the filtration

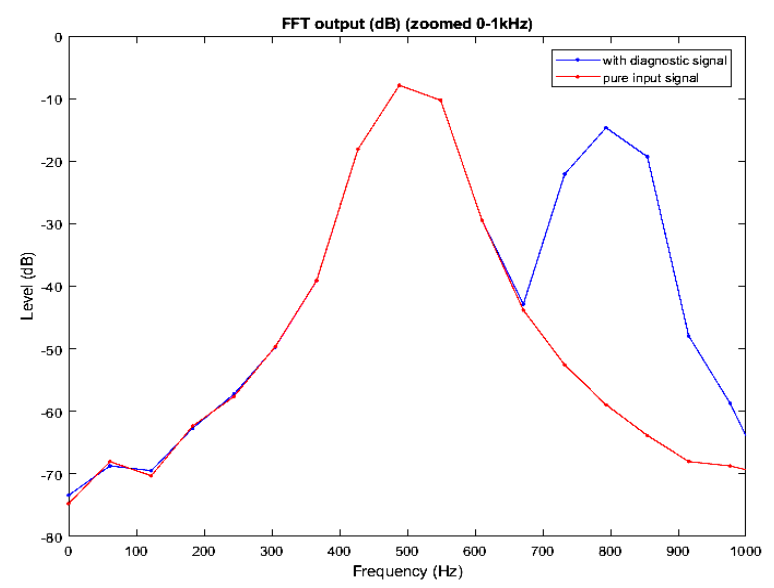

Fig. 9. The calculated spectrum density, with the help of the model on the FFT algorithm
The calculation results of the spectral density in accordance with the Goertzel's algorithm:

$\checkmark$ Block №1, harmonic № 66 (frequency $=503.54 \mathrm{~Hz}$ ), respond without diagnostic $=0.3398$, respond with diagnostic $=0.3404$.

$\checkmark$ Block №2, harmonic № 67 (frequency $=511.17 \mathrm{~Hz}$ ), respond without diagnostic $=0.1952$, respond with diagnostic $=0.1946$.

$\checkmark$ Block №3, harmonic № 104 (frequency $=793.46 \mathrm{~Hz}$ ), respond without diagnostic $=0.0036$, respond with diagnostic $=0.0275$.

$\checkmark$ Block №4, harmonic № 105 (frequency $=801.09 \mathrm{~Hz}$ ), respond without diagnostic $=0.0035$, respond with diagnostic $=0.1830$.

Alternatively, in MATLAB there was made the calculation of the spectrum density on the signal rolling frequencies and the signal self-control with turned off and on self-control system. The calculation results on FFT are $-15 \mathrm{~dB}$ at $800 \mathrm{~Hz}$ frequency, on the Goertzel's algorithm block №4, $20 \mathrm{log}$ (0.183) - $15 \mathrm{~dB}$, values match [13-18].

\section{DISCUSSION}

The spheres of the usage of the vibration-diagnostic systems with the metrological control function are very wide; they are any vibrating machines and mechanisms, which condition demands the constant control, the transport, the machine tools.

One of the most probable spheres of the usage of the given system is the diagnostic of the rolling bearings, the wheel gear units on the railway transport.

The proposed method in the contradiction to the other known methods $[19,20]$, allows the flexibly reconfiguration of as well as the frequency of a self-control system signal depending on the sphere of the usage of the vibration diagnostic system as the activation time of the self-control system with the continuous, periodic or adaptive self-control.

Some research results can be used in other disciplines, for example, chemistry and ecology [2124]. They have a significant impact not only on the chemical composition of the atmosphere, hydrosphere and lithosphere, but also on the ecosystem [25].

The effectiveness of the case study for developing of the environmental accountability of engineers is proved by the choice of the case - the description of the ecological challenges and other parameters [26]. It is assumed that the proposed method of metrological self-control will help to implement such functions.

\section{CONCLUSION}

In the article there is the review of existing elemental base for creating the vibration diagnostic systems for the different purposes. The review includes the recommendations on the choice of the sensitive element, the analog-to-digital converter, the microcontroller input amplifier, the communication interface. There is given the description of the vibration signal analysis methods and there is given the most detailed method of the self-diagnostic of the whole measuring path. 


\section{Measuring System of Vibration Diagnostic with the Metrological Self-Control Function}

The structure of the integrated microcircuit "VIBRO-ASIC", that is the basic component of the vibration diagnostic system, is defined. There are shown the principles of the realization of the given method of the self-diagnostic in the microcircuit. The experiment for the estimation of the efficiency of the proposed method was conducted

There are shown the advantages of the integral performance of this system compared with the traditional diagnostic methods. There are reviewed the main spheres of the usage.

\section{ACKNOWLEDGMENT}

The given work was made on support of the Ministry of Education and Science of Russian Federation on the Agreement about the providing of the subsidies №14.581.21.0030 from the $27^{\text {th }}$ of October 2017, the unique identification of the work (project) is RFMEFI58117X0030.

\section{REFERENCES}

1. R.E. Taimanov, and K.V. Sapozhnikova. "Metrological self-monitoring of sensors", Datchiki i sistemy, 2, 2011, pp. 58-66.

2. A.N. Pronin, K.V. Sapozhnikova, and R.E. Taimanov. "Monitoring the accuracy of information received from sensors", Datchiki $i$ sistemy, 8 , 2008, pp. 58-63.

3. Yu. Ponomariov. "Colibrys: Modern technologies with Swiss quality", Komponenty i tekjnologii, 10, 2015, pp. 34-38.

4. Vibration sensor 4-20 MA Output velocity sensor. Retrieved from: https://www.pcb.com/contentstore/docs/PCB_Corporate/IMI/Products/ Specsheets/640B11_B.pdf (access date: 16.10.2019).

5. Digital Tri-Axial Vibration Sensor. Accelerometer ADIS16223. Retrieved from: https://www.analog.com/media/en/technical-documentation/data-sheet s/adis16223.pdf (access date: 16.10.2019).

6. Description of Accelerometer ALE-049M. Retrieved from: www.all-pribors.ru/docs/62710-15.pdf (access date: 15.10.2019).

7. S. Sysoeva. "Key segments of MEMS market. Accelerometers", Komponenty i tekhnologii, 2, 2010, pp. 20-26.

8. Yu. Ponomariov. "The choice of vibration accelerometers: a simple solution to a complex problem", Elementarnaya baza elektroniki, 2(00142), 2015, pp. 116-120.

9. $1 \mathrm{nV} / \sqrt{\mathrm{Hz}}$ Low Noise $210^{\circ} \mathrm{C}$ Instrumentation Amplifier. AD8229. Retrieved

from: https://www.analog.com/media/en/technical-documentation/data-sheet s/AD8229.pdf (access date: 17.10.2019).

10. $500 \mathrm{MHz}$, Linear-in-dB VGA with AGC Detector. AD8367. Retrieved from:

https://www.analog.com/media/en/technical-documentation/data-sheet s/AD8367.pdf (access date: 17.10.2019).

11. Micropower, Single- and Dual-Supply, Rail-to-Rail Instrumentation Amplifier. AD627. Retrieved from: https://www.analog.com/media/en/technical-documentation/data-sheet s/AD627.pdf (access date: 16.10.2019).

12. Precision Instrumentation Amplifier. AD8221. Retrieved from: https://www.analog.com/media/en/technical-documentation/data-sheet S/AD8221.pdf (access date: 14.10.2019).

13. S. Devendiran, and K. Manivannan. "Vibration based condition monitoring and fault diagnosis technologies for bearing and gear components: A review", International Journal of Applied Engineering Research, 11(6), 2016, pp. 3966-3957

14. V.N. Kostyukov, A.P. Naumenko, S.N. Bojchenko, and K. Tarasov. Fundamentals of vibro-acoustic diagnostics of machinery. Omsk: NPTS "Dynamics" Press, 2007. 286 p.

15. V.F. Gorbunov, and G.S. Zhartovski. "On the parameter characterizing the size of dynamic frequency range of vibration sensors", Herald of the Tomsk Polytechnic University, 2, 2008, pp. 128-134.

16. Yu.I. Majer, and A.I. Kalinichenko. "Dependence of vibration sensors on mounting methods", Herald of the Tomsk Polytechnic University, 1, 2016, pp. 322-325.
17. M.D. Genkin, and A.G. Sokolova. Vibroacoustic diagnostics of machines and mechanisms. Moscow: Mashinostroenie Press, 1987. 190 p.

18. M. Karimi. Rolling element bearing Fault diagnostics using the blind deconvolution: $\mathrm{PhD}$ thesis. Queensland University of Technology, 2006.

19. GOST R 8.734-2011. State system for ensuring the uniformity of measurements. Intelligent sensors and intelligent measuring systems. Methods of metrological self-control. Retrieved from: http://docs.cntd.ru/document/gost-r-8-734-2011-gsi (access date: 10.10.2019).

20. GOST 25275-82 System of standards for vibration. Retrieved from: http://docs.cntd.ru/document/1200023547 (access date: 10.10.2019).

21. N.S. Pshchelko, E.G. Vodkailo, V.V. Tomaev, B.D. Klimenkov, V.L. Koshevoi, and A.O. Belorus. "Influence of electric field on adhesion and structure of conducting films on dielectric substances", Izvestija vysshykh uchebnykh zavedenij. Serija "Khimija $i$ khimicheskaja tekhnologija”, 60(8), 2017, pp. 100-104. DOI: 10.6060/tcct.2017608.5649

22. A. Strizhenok., D. Korelskiy. "Assessment of the state of soil-vegetation complexes exposed to powder-gas emissions of nonferrous metallurgy enterprises", Journal of Ecological Engineering, 17(4), 2016, pp. 25-29. DOI: 10.12911/22998993/64562

23. V.S. Talismanov, S.V. Popkov, O.G. Karmanova, S.S. Zykova. "Synthesis and fungicidal activity of substituted 1-[(1,3-dioxolan-4-yl)]methyl-1H-imidazoles and 1-[(1,3-dioxolan-4-yl)methyl]-1H-1,2,4-triazoles based on arylidene ketones", International Journal of Pharmaceutical Research, 11(2), 2019, pp. 315-319. DOI: 10.31838/ijpr/2019.11.02.051

24. K.S. Kupavykh, and A.V. Shipulin. "Pulsed method of coalbed methane drainage", Gornyi Zhurnal, 5, 2018, pp. 78-82. DOI: 10.17580/gzh.2018.05.12

25. A. Strizhenok, and D. Korelskiy. "Assessment of the anthropogenic impact in the area of tailings storage of the apatite-nepheline ores", Pollution Research, 34(4), 2015, pp. 809-811.

26. I.V. Goman. "Case study analysis as a way of developing the environmental accountability of future oil and gas engineers", International Multidisciplinary Scientific GeoConference Surveying Geology and Mining Ecology Management, SGEM 2017, 17(54), 2017, pp. 17-26. 